\title{
New Technique for Static Target Detection in Dense-Multipath Urban Environments
}

\author{
Imanol de Arriba-Ruiz, Félix Pérez-Martínez \\ Department of Signals, Systems and \\ Radiocommunications. Technical University of Madrid \\ Avda. Complutense s/n, 28040 \\ Madrid, Spain \\ \{imanol, felix\}@gmr.ssr.upm.es
}

\author{
José María Muñoz-Ferreras \\ Department of Signal Theory and \\ Communications. University of Alcalá \\ Ctra. Madrid-Barcelona, 28805 \\ Alcalá de Henares, Madrid, Spain \\ jm.munoz@uah.es
}

\begin{abstract}
Synthetic Aperture Radar (SAR) images a target region reflectivity function in the multi-dimensional spatial domain of range and cross-range with a finer azimuth resolution than the one provided by any on-board real antenna. Conventional SAR techniques assume a single reflection of transmitted waveforms from targets. Nevertheless, new uses of Unmanned Aerial Vehicles (UAVs) for civilian-security applications force SAR systems to work in much more complex scenes such as urban environments. Consequently, multiple-bounce returns are additionally superposed to direct-scatter echoes. They are known as ghost images, since they obscure true target image and lead to poor resolution. All this may involve a significant problem in applications related to surveillance and security. In this work, an innovative multipath mitigation technique is presented in which Time Reversal (TR) concept is applied to SAR images when the target is concealed in clutter, leading to TR-SAR technique. This way, the effect of multipath is considerably reduced -or even removed-, recovering the lost resolution due to multipath propagation. Furthermore, some focusing indicators such as entropy $(E)$, contrast $(C)$ and Rényi entropy $(R E)$ provide us with a good focusing criterion when using TRSAR.
\end{abstract}

Index Terms - Time Reversal (TR), Synthetic Aperture Radar (SAR), multipath, ghost, focusing, entropy, contrast, Rényi entropy.

\section{INTRODUCTION}

The name of the technique -Synthetic Aperture Radar $(S A R)$ - alludes to the concept of signal synthesis, which offers an improvement in azimuth resolution [1]-[4]. At the same time, a comparable range resolution is also required. For this purpose, it is very common to employ linear frequency modulated (chirped) pulses in order to use compression techniques in reception.

The use of centimetric and millimetric wavelengths turns the SAR technique into an 'all-weather' system since it is able to work under adverse weather conditions and it has a day/night capability to obtain images. Nevertheless, there are other effects that SAR techniques are not able to face up to so efficiently, which are connected with a series of internal and external factors to the SAR system -among them, multipath effect, the well-known speckle noise or phase noise must be

This work has been supported by Project TEC2008-02148 of the Spanish National Board of Scientific and Technology Research. mentioned. In consequence, images are declined in terms of quality and resolution.

The purpose of our previous work was to model and simulate scenes affected by multipath [5]. Typical SAR systems are designed for imaging targets with a direct line of sight of the SAR sensors. Nonetheless, in environments where the intended target is hidden or surrounded by many scatterers, its signature consists of the directly reflected waveform as well as the secondary returned waveforms due to those scatterers, which severely degrade image resolution [6].

In this paper, an innovative multipath mitigation technique is presented in which Time Reversal (TR) concept is applied to SAR images when the target is concealed in clutter, leading to $T R$-SAR technique [7]-[8]. Our motivation comes from the fact that most of the techniques aimed at reducing multipath, quoted in the literature, are related to antenna design. For this reason, we believe in the need to develop or improve signal processing techniques focused on multipath mitigation.

In a nutshell, from an estimated range of the prominent points of a conventional SAR image riddled with multipath, TR-SAR is able to focus on target scatterers with a finer resolution, reducing (or even removing) ghost artifacts. For this purpose, this algorithm processes phase history data using the multi-path response of a dense-clutter environment constructively.

That is the reason why TR-SAR is used as a method for detecting the presence of static targets, specifically in highly cluttered environments. By exploiting these strengths, a TRSAR system to monitor forests or dense urban environments is deployed, increasing the probability of detecting a target [9]. In short, its ultimate goal is to improve automated target recognition in dense multipath [7]. To that end, focusing indicators can be really useful [10]-[12], as it will be shown in this work.

\section{TIME REVERSAL (TR)}

Multipath is usually thought to be detrimental and its effects should be minimized. Time Reversal presents the opposite opportunity -multipath is seen as a positive, the more the better [13]. In fact, TR is essentially a multipath based technique since it takes advantage of the scattering and the temporal diversity induced by multipath in inhomogeneous channels in order to increase resolution. Interestingly, if the multipath is rich enough, the channel itself does focus the time reversed signal back on the original source, reconstructing the original transmitted signal approximately [14]. 
An important drawback when it comes to mitigating multipath is the fact that there is not a priori information about scatterers, such as their actual position or their physical features. As a result, these kinds of techniques have no alternative but to work blindly with having no knowledge of geometry. It causes that distinguishing true targets from ghost images can be a very difficult task since the only information is a conventional SAR image corrupted by multipath. Once a target spot is identified, it is possible to examine its shape or its size with improved resolution.

Not only has TR been used to focus selectively on different scatterers in a medium, but it has also been really useful in the field of detection. In that context, TR can be regarded as an adaptive waveform transmission technique, since it matches the transmitted waveform to the channel. For this reason, TR avoids the detailed modelling of the channel, providing a gain from matching to the propagated field. In a sense, TR provides the actual channel Green's function [9], [14]-[15]. It is worth noting that TR requires a coherent processing of the data before image formation algorithms are applied in order to optimize signal-to-noise ratio $(S N R)$ and detection probability [9].

\section{MATHEMATICAL DESCRIPTION OF TR-SAR}

\section{A. Signal Model}

The transmitted signal is wide-band -linear frequency modulated (LFM) pulses-, whose instantaneous frequency increases with time at a rate of $\gamma$ (chirp rate):

$$
f_{\text {inst }}=f_{c}+\gamma \cdot(t-n \cdot T)
$$

where $f_{c}$ is the central operational frequency, $t=n \cdot T$ represents the center point of the $n$-th pulse ( $n=0$ corresponds with the first transmitted pulse), and $\gamma$ is given by the quotient between the transmitted bandwidth $(B)$ and the inter-pulse period $(T)$.

On the other hand, the SAR system transmits LFM pulses at a rate of PRF (Pulse Repetition Frequency), which corresponds to the inverse of $T$. The hypothesis of stop and go has been made, that is, the distance covered by the radar, between transmission and reception of each pulse, is considered to be negligible (intra-pulse motion is null). This way, the radar motion is assumed to be discrete, so transmission and reception will be carried out when the SAR system is stopped.

The expression of each transmitted LFM pulse is given by:

$$
s_{t}(n, t)=\operatorname{rect}(\hat{t} / T) \cdot \cos \left(2 \pi f_{c} t+\pi \hat{t}^{2}\right),
$$

where $\operatorname{rect}(\cdot)$ is the rectangular window function and $\hat{t}=t-n \cdot T$ is known as fast-time, that is, the time in which each pulse is received.

In SAR context, signal phase is far more important than amplitude. For this reason, mathematical analysis requires the use of the complex-valued form of the signal.

$$
s_{t}(n, t)=\operatorname{rect}(\hat{t} / T) e^{j\left(2 \pi f_{c} t+\pi \hat{\varkappa}^{2}\right)} .
$$

\section{B. Configuration}

As far as configuration is concerned, it is a mono-static system, which is illustrated in Fig. 1. The SAR sensor is onboard an aircraft at height $z=h, x$ and $y$ are cross-range and ground-range coordinates, respectively, $\psi$ is the grazing angle, $L$ represents the synthesized aperture length and $W$ is the swath width.

Unlike [7] and [8], it is important to emphasize that our TRSAR algorithm has been developed for stripmap mode [1]. The reason is that the chosen SAR image formation algorithm -RMA, Range Migration Algorithm- requires azimuth chirp preservation so as to work properly.

Another distinction with regard to the contribution of the authors of [7] and [8] is the fact that they carry out two-pass data collections of the same scene in order to detect targets. The difference between them is that the first one is made when targets are not present so as to characterize the clutter environment. This way, subtracting out this measurement from the second step one, it is possible to remove most of the targets surrounding scatterers contribution.

Nevertheless, in our case, only one scanning of the analyzed scene has been accomplished, making the assumption that the clutter level is not so high as the one of the direct echoes coming from the desired targets. We consider that this is a more realistic case since in most of real scenes it is not possible to extract, and later insert, the desired targets. That is the reason why the received signal will contain direct echoes as well as multiple-bounce returns not only due to the desired targets but also owing to the scatterers located in their vicinity -an exhaustive characterization of multipath was developed in our previous work [5].

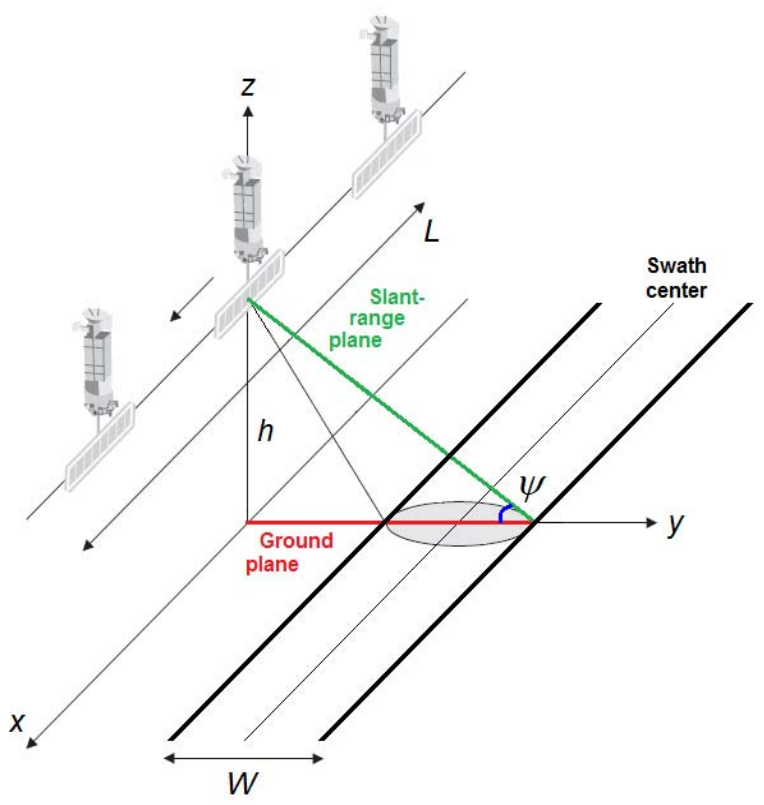

Fig. 1. Stripmap SAR: Mono-static system 


\section{TR-SAR Algorithm}

As it was introduced in Section II, TR is able to turn multipath propagation into a desirable effect. Broadly speaking, TR-SAR algorithm processes multipath corrupted phase history data following the next stages [7]-[9], [13]-[15], which will be repeated for each detected prominent point $(k=1, \ldots, K)$ and for each transmitted pulse $(l=1, \ldots, L)$ :

1) Time Reversal: The received signal coming from the $k$-th prominent point will be made up of the direct echo (denoted by subscript $d$ ) and an undefined number $(m=1, \ldots, M)$ of multipath returns (denoted by subscript $r$ ):

$$
\begin{aligned}
& s_{r}(n, t)=s_{d}(n, t)+\sum_{m=1}^{M} s_{r m}(n, t)=\sigma_{k} \cdot \operatorname{rect}\left(\left(\hat{t}-t_{d}\right) / T\right) \\
& \cdot\left[e^{j 2 \pi f_{c}\left(t-t_{d}\right)} \cdot e^{j \pi \gamma\left(\hat{t}-t_{d}\right)^{2}}+\sum_{m=1}^{M}\left|\Gamma_{s m}\right|^{2} \cdot e^{j 2 \pi f_{c}\left(t-t_{r m}\right)} \cdot e^{j \pi \gamma\left(\hat{t}-t_{r m}\right)^{2}}\right],
\end{aligned}
$$

where

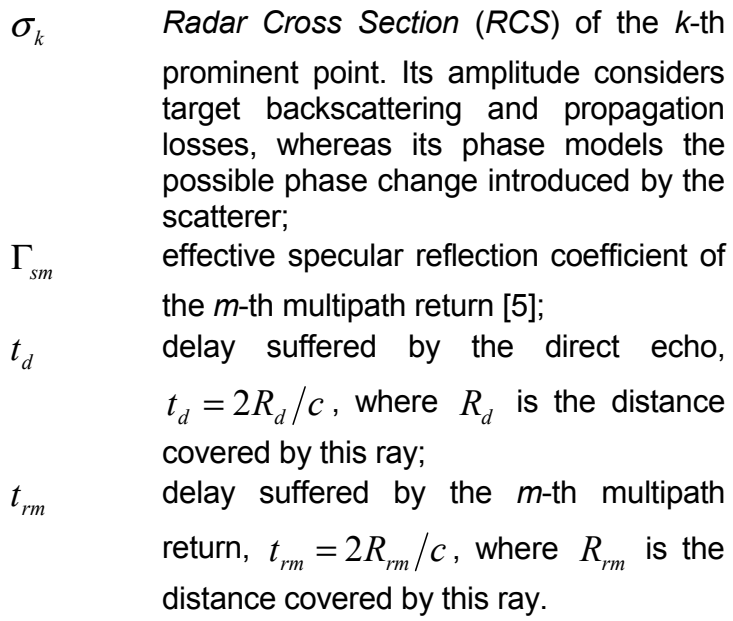

This signal is stored and delayed by a sufficiently large delay ( $T_{c}$, the chosen time window length):

$$
s_{r}(n, t) \underset{\text { delay }}{\longrightarrow} s_{r}\left(n, t-T_{c}\right)
$$

Then, it is time-reversed, which in the frequency domain corresponds to phase conjugation [16]:

$$
\begin{gathered}
s^{T R}(n, t)=s_{r}\left(n, T_{c}-t\right) \stackrel{ }{\longrightarrow F T_{t}} \\
S^{T R}(n, \omega)=e^{j \omega T_{c}} \cdot S_{r}^{*}(n, \omega) .
\end{gathered}
$$

A linear phase term arises due to this delay -phase shift $e^{j \omega T_{c}}-$, which can be regarded negligible:

$$
S^{T R}(n, \omega) \approx S_{r}^{*}(n, \omega)
$$

2) Energy Normalization: The signal is energy normalized. To that end, it is multiplied by a constant which is given by:

$$
z=\sqrt{\frac{\int\left|s_{t}(n, t)\right|^{2} d t}{\int\left|s_{r}(n, t)\right|^{2} d t}} .
$$

Therefore, the obtained signal before accomplishing retransmission is:

$$
\begin{gathered}
s_{t}^{T R}(n, t)=z \cdot S^{T R}(n, t)=z \cdot \sigma_{k}^{*} \cdot \operatorname{rect}\left(\left(\hat{t}-t_{d}\right) / T\right) \\
\cdot\left[e^{-j 2 \pi f_{c}\left(t-t_{d}\right)} \cdot e^{-j \pi \gamma\left(\hat{t}-t_{d}\right)^{2}}+\sum_{m=1}^{M}\left|\Gamma_{s m}\right|^{2} \cdot e^{-j 2 \pi f_{c}\left(t-t_{r m}\right)} \cdot e^{-j \pi \gamma\left(\hat{t}-t_{r m}\right)^{2}}\right]
\end{gathered}
$$

3) Signal Retransmission: The resulting signal is mathematically retransmitted back to the target area through the same channel:

$$
\begin{gathered}
s_{r}^{T R}(n, t)=z \cdot\left|\sigma_{k}\right|^{2} \cdot \operatorname{rect}\left(\left(\hat{t}-t_{d}\right) / T\right) \\
\left.\left.\cdot\left|e^{j 2 \pi f_{c}\left(t-t_{d}\right)} \cdot e^{j \pi \gamma\left(\hat{t}-t_{d}\right)^{2}}+\sum_{m=1}^{M}\right| \Gamma_{s m}\right|^{2} \cdot e^{j 2 \pi f_{c}\left(t-t_{r m}\right)} \cdot e^{j \pi \gamma\left(\hat{t}-t_{r m}\right)^{2}}\right|^{2}
\end{gathered}
$$

According to (10), it is possible to carry out signal retransmission by simply calculating the square of the absolute value of the signal (9). This way, it has been demonstrated that it is possible to accomplish the developed algorithm without having to reradiate the received signal into the original medium physically, which would really complicate its implementation in a real system.

4) Focusing Process: Finally, a focusing process is carried out in order to reconstruct the image -TR-SAR dechirping. To that end, it is essential to know the estimated range of the prominent points of a conventional SAR image corrupted by multipath. This way, TR-SAR is able to focus on these dominant patterns -on the desired target scattererswith a finer resolution than conventional techniques. As a result, the effect of multipath is considerably reduced, or even removed.

Nevertheless, if the focused spot is not a target, the details will be blurred and random. For this reason, it is very important to develop an algorithm capable of detecting image prominent points accurately by searching the spots that exceed a threshold. Those dominant patterns will correspond with the direct echoes coming from the desired target scatterers since we have to bear in mind that multipath returns suffer more attenuation than direct echoes, not just because they cover a larger distance but because they also lose part of their power when being incident on a certain surface; for this reason, their amplitude tend to be lower than the direct echoes.

In order to accomplish TR-SAR dechirping, it is necessary to multiply $s_{r}^{T R}(n, t)$ by the conjugate of the following reference signal:

$$
s_{r e f}^{T R}(n, t)=\left[\sum_{k=1}^{K} e^{-j 2 \pi f_{c}\left(t-\tilde{t}_{k}\right)} \cdot e^{-j \pi \gamma\left(\hat{t}-\tilde{t}_{k}\right)^{2}}\right]
$$




$$
\cdot\left[e^{j 2 \pi f_{c}\left(t-t_{o}\right)} \cdot e^{j \pi \gamma\left(\hat{t}-t_{o}\right)^{2}}\right]
$$

where

$\widetilde{t}_{k} \quad$ is given by $\widetilde{t}_{k}=2 \widetilde{R}_{k} / c$, being $\widetilde{R}_{k}$ the estimated distance between the radar and the $k$-th prominent point;

$t_{o} \quad$ is given by $t_{o}=2 R_{o} / c$, where $R_{o}$ is the reference distance. In stripmap mode, it represents the distance between the radar and the swath centre;

According to (11), this signal is composed of two terms. The first one serves to focus on the estimated ranges of the prominent points. That is the reason why this term is made up of the addition of as many components as the number of the detected prominent points. The second one centres the reconstructed image at the distance indicated by $R_{o}$.

That being said, the final SAR image free of multipath is given by:

$$
s_{\text {final }}(n, \hat{t})=s_{r}^{T R}(n, t) s_{\text {ref }}^{T R^{*}}(n, t) .
$$

To sum up, only prominent points are desired to appear in the reconstructed SAR image. To that end, TR-SAR algorithm processes phase history data using the multi-path response of a dense-clutter environment constructively. Interestingly, if the scattering channel is reciprocal and highly dispersive -rich in multipath-, the channel itself does focus the time reversed signal back on the original source, reconstructing the original transmitted signal approximately.

\section{RESULTS}

\section{A. Definitions}

Multipath phenomenon can be very pernicious since ghost echoes can mask true targets. Nonetheless, the existence of any feature which allows us to distinguish direct-scatter returns from ghost artifacts will sort out the problem. In [5], some parameters such as QPE (Quadratic Phase Error), ISLR (Integrated Sidelobe Ratio) and PSLR (Peak Sidelobe Ratio) provided us with the tools to achieve our goal. However, in this work, a rich scattering environment with many reflected rays, as well as direct returns, is simulated. In this kind of cases in which returns are so close, secondary lobes -and even main lobes- tend to interfere with each other. In consequence, the resulting values of QPE, ISLR and PSLR will be erroneous. For this reason, we have inclined to use focusing indicators such as Entropy $(E)$, Contrast $(C)$ and Rényi Entropy $(R E)$ of order $\alpha=0.5$ [10]-[12]:

$$
\begin{gathered}
E=-\sum_{i} \sum_{j} \bar{I}_{i, j} \cdot \ln \bar{I}_{i, j}, \\
C=\frac{\sqrt{A\left\{\left[\left|I_{i, j}\right|^{2}-A\left(\left|I_{i, j}\right|^{2}\right)\right]^{2}\right\}}}{A\left(\left|I_{i, j}\right|^{2}\right)},
\end{gathered}
$$

$$
R E=\frac{1}{1-\alpha} \ln \left(\sum_{i} \sum_{j} \bar{I}_{i, j}^{\alpha}\right),
$$

where $I_{i, j}$ is the SAR image, $i$ and $j$ are the spatial coordinates, $A(\cdot)$ operator represents the spatial mean and $\bar{I}_{i, j}$ is the normalized SAR image, given by the expression:

$$
\bar{I}_{i, j}=\frac{\left|I_{i, j}\right|^{2}}{\sum_{i} \sum_{j}\left|I_{i, j}\right|^{2}} .
$$

As for multipath features, the rays are reflected from a smooth surface -specular multipath- using a flat Earth model. Nevertheless, they are assumed to be back-scattered from each of the incidence points and to cover, in its return path, the same one as they covered until reaching the target in its outward path. In other words, they do not verify Snell's law and, to a certain extent, a diffuse behaviour is thus imitated. Otherwise, only one reflected ray could be taken into account.

\section{B. Simulations Results}

In order to form SAR images of scenes affected by multipath, one of the most efficient SAR image formation algorithms has been used $-R M A$. Its ability to compensate range curvature accurately must be highlighted. The most relevant simulation parameters of the used radar are: central operational frequency $(242.2 \mathrm{MHz})$, transmitted bandwidth (133.5 MHz), PRF (320 Hz) and sampling rate $(192 \mathrm{MHz})$.

Our contribution consists in demonstrating TR-SAR robustness. For this purpose, a scene made up of 3 point targets located at positions [0m, 0m, 100m], [-200m, 0m, 100m] and $[-200 \mathrm{~m}, 200 \mathrm{~m}, 100 \mathrm{~m}]$ has been created. Each one has 20 associated multipath echoes as well as the direct return (urban environment). The resulting SAR image is depicted in Fig. 2. It is worth noting that these targets are elevated above the background terrain. As a result, they appear a bit shifted in slant-range direction due to their projection on the slant-range plane [1]. If they were not elevated, the spots would appear in the aforementioned positions.

As it was previously mentioned, focusing process is an essential step in TR-SAR so as to reconstruct the image. Fig. 3 depicts the obtained image after focusing exactly on the desired targets -this corresponds with the case in which the prominent points of the conventional SAR image corrupted by multipath have been accurately detected. The efficiency of this algorithm is obvious when it is used properly, since there is no doubt when it comes to distinguishing the true targets in the image. In other words, the lost resolution due to multipath propagation has been recovered. Even in the case of having two of the three targets in the same azimuth position $(-200 \mathrm{~m})$, in which the echoes of the left-hand side target mask the righthand side target, TR-SAR is able to provide excellent results.

On the other hand, if the detection of the prominent points is inaccurate, the focusing process will not be properly accomplished -this could happen when a high level spot of the image is mistaken for a desired point target. In Fig. 4, TRSAR algorithm has focused on positions $[100 \mathrm{~m},-100 \mathrm{~m}, 100 \mathrm{~m}]$, $[-100 m,-100 m, 100 m]$ and $[-100 m, 100 m, 100 m]$, which do not 
match the ideal ones. The more inaccurate the detection is, the worse quality image will have (the targets level tends to be a bit lower and their resolution gets slightly worse), as it is shown in Fig. 5, where more inaccurate positions have been used to carry out the focusing process: [250m,-250m,100m], $[50 \mathrm{~m},-250 \mathrm{~m}, 100 \mathrm{~m}]$ and $[50 \mathrm{~m},-50 \mathrm{~m}, 100 \mathrm{~m}]$.

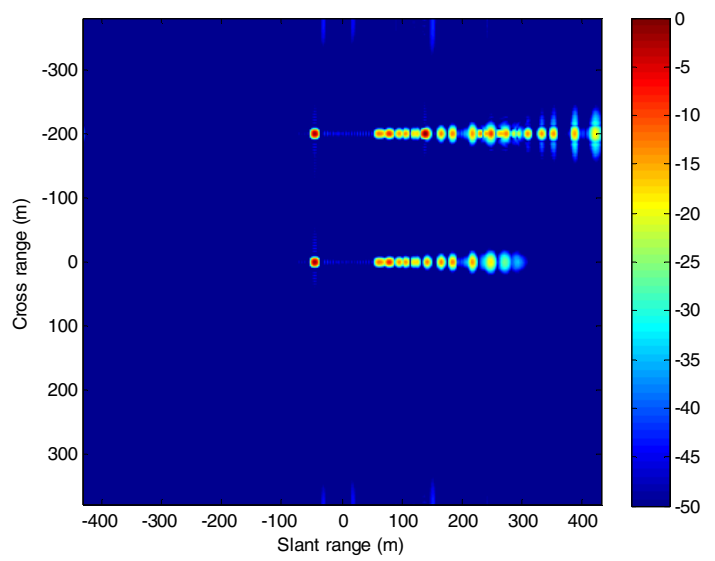

Fig. 2. Three point targets with 20 associated multipath echoes

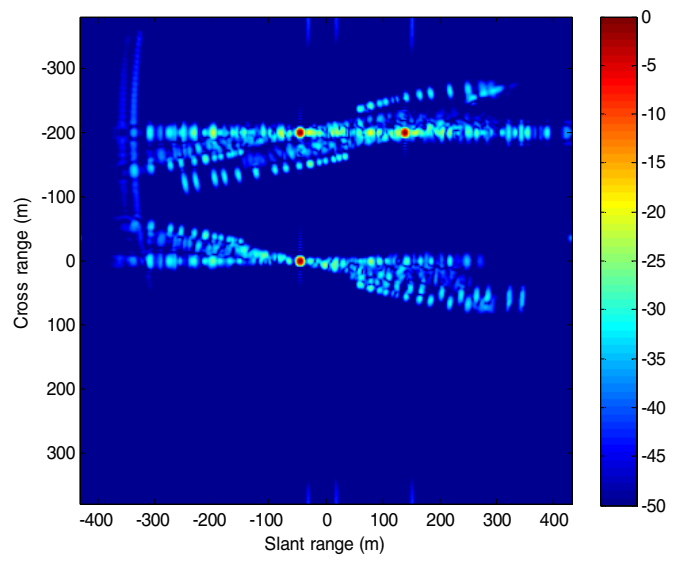

Fig. 3. Image after TR-SAR: Focusing exactly on the desired point targets

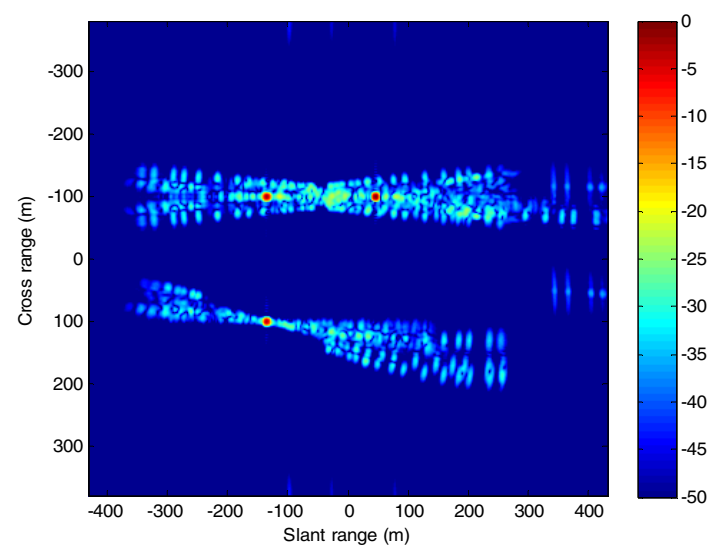

Fig. 4. Image after TR-SAR: Not focusing on the desired point targets $(\mathrm{I})$

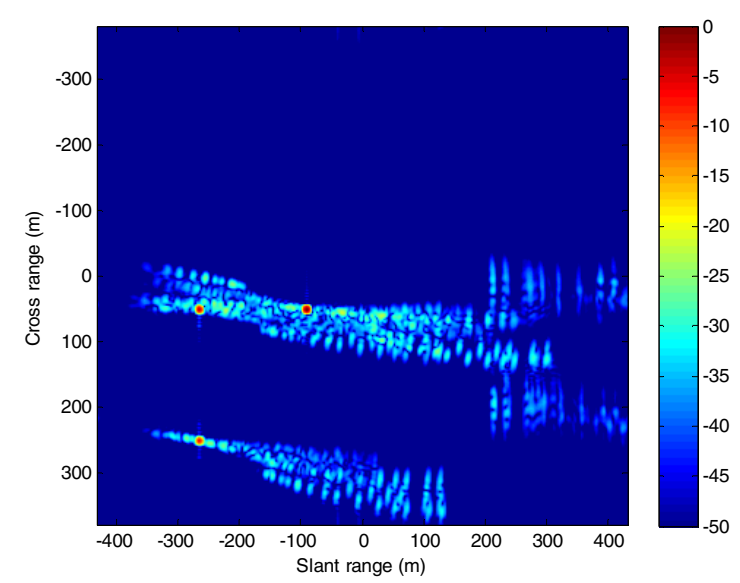

Fig. 5. Image after TR-SAR: Not focusing on the desired point targets (II)

By analyzing the focusing indicators, it is possible to verify that, as the detection of the prominent points is more inaccurate, most of these parameters get worse (Table 1). In conclusion, these indicators provide us with a good focusing criterion when using TR-SAR. For example, in a situation in which there are several dominant patterns but only one of them corresponds to the true point target, many doubts may arise when it comes to choosing which one corresponds with the intended target. In this case, it is needed to focus on each of them and the one which provides the minimum image entropy after applying TR-SAR will be the desired point target.

In fact, by analyzing how entropy evolves before and after deploying TR-SAR (Table 1), we can state that this is the most reliable parameter. When the image is corrupted by multipath, $E$ is higher than after applying TR-SAR. Indeed, the best value of $E$ takes place when focusing exactly on the desired targets. In other words, the more inaccurate the prominent points detection is, the bigger $E$ will be. As for contrast, after focusing in TR-SAR dechirping accurately, its value gets considerably better. Nonetheless, it provides a confusing result when focusing inaccurately since it tends to increase but it sometimes decreases, whose interpretation is not obvious at all. Finally, Rényi entropy makes worse a lot when focusing exactly on the desired targets, but it also increases as the prominent points detection is more inaccurate. It is very helpful so as to focus better, and it is considered to be a piece of additional information that contrast is not able to provide us.

TABLE I

EVOLUTION OF FOCUSING INDICATORS

\begin{tabular}{|c|c|c|c|}
\hline & $E$ & $C$ & $R E$ \\
\hline $\begin{array}{c}\text { Image with multipath before } \\
\text { TR-SAR (Fig. 2) }\end{array}$ & 7.717 & 31.427 & 9.136 \\
\hline $\begin{array}{c}\text { After TR-SAR with accurate } \\
\text { focusing (Fig. 3) }\end{array}$ & 7.263 & 45.758 & 10.020 \\
\hline $\begin{array}{c}\text { After TR-SAR with inaccurate } \\
\text { focusing (I) (Fig. 4) }\end{array}$ & 7.440 & 46.499 & 10.157 \\
\hline $\begin{array}{c}\text { After TR-SAR with inaccurate } \\
\text { focusing (II) (Fig. 5) }\end{array}$ & 7.583 & 50.042 & 10.358 \\
\hline
\end{tabular}




\section{v. CONCLUSIONS}

In this article, the efficiency of TR when it is applied to SAR images in rich multipath environments has been shown. The most noteworthy fact is that TR-SAR behaves like an amplitude modulation $(A M)$. In the case of considering a single reflected ray, the carrier would be equivalent to the direct echo -the dominant pattern where TR-SAR focuses on- and the sidebands to the multipath echo. When there are many multipath echoes, the modulation structure is much more variable -its envelope has a noisy appearance. Therefore, although the contribution of sidebands is wider than in the previous case, their level is lower -its spectrum is wide-band and it seems that they have been averaged. In SAR context, this means that the multipath contribution is clearly reduced. That is the reason why TR-SAR provides better results in a rich multipath environment. Furthermore, the developed algorithm can be extrapolated to an extended target, which will be made up of a cluster of point reflectors. Finally, it is worth noting that entropy, contrast and Rényi entropy provide us with a good focusing criterion when using TR-SAR.

\section{REFERENCES}

[1] W. G. Carrara, R. S. Goodman, and R. M. Majewski, Spotlight Synthetic Aperture Radar. Signal Processing Algorithms, Artech House, 1995.

[2] C. A. Wiley, "Synthetic Aperture Radars", IEEE Trans. on Aerospace and Electronic Systems, vol. AES-21, no. 3, pp. 440-443, May 1995.

[3] D. L. Mensa, High Resolution Radar Imaging, Norwood, MA, Artech House, 1981.

[4] I. G. Cumming, and F. H. Wong, Digital Processing of Synthetic Aperture Radar Data. Algorithms and Implementation, Artech House, 2005.

[5] I. de Arriba-Ruiz, F. Pérez-Martínez, and J. M. MuñozFerreras, "Multipath Reflectivity Estimation in Urban Environments for Synthetic Aperture Radar Images", Military Communications Conference (MILCOM 2010), pp. 864-869, San Jose, California, November 2010.

[6] Y. Jin, J. M. F. Moura, and N. O'Donoughue, "Experimental Study of Extended Target Imaging by Time Reversal SAR", IEEE International Conference on Acoustics, Speech and Signal Processing (ICASSP '09), pp. 2109-2112, Taipei, Taiwan, April 2009.

[7] Y. Jin, J. M. F. Moura, M. T. Mulford, N. O'Donoughue, and A. Samuel, "Time Reversal Synthetic Aperture Radar Imaging in Multipath", Proc. 41st Annual Asilomar Conference on Signals, Systems, and Computers (Asilomar '07), pp. 1812-1816, Pacific Grove, CA, November 2007.

[8] Y. Jin, and J. M. F. Moura, "TR-SAR: Time Reversal Target Focusing in Spotlight SAR", IEEE International Conference on Acoustics, Speech and Signal Processing (ICASSP '07), pp. 957-960, Honolulu, Hawaii, April 2007.

[9] N. O'Donoughue, J. M. F. Moura, and Y. Jin, "SignalDomain Registration for Change Detection in TimeReversal SAR", Proc. 42nd Annual Asilomar Conference on Signals, Systems, and Computers (Asilomar '08), Pacific Grove, CA, October 2008.
[10] L. Xi, L. Guosui, and J. Ni, "Autofocusing of ISAR Images based on Entropy Minimization", IEEE Transactions on Aerospace and Electronic Systems, vol. 35, no. 4, pp. 1240-1252, October 1999.

[11] F. Berizzi, and G. Corsini, "Autofocusing of Inverse Synthetic Aperture Radar Images using Contrast Maximization", IEEE Transactions on Aerospace and Electronic Systems, vol. 32, no. 3, pp. 1185-1191, July 1996.

[12] J. M. Muñoz-Ferreras, F. Pérez-Martínez, and M. Datcu, "Generalisation of Inverse Synthetic Aperture Radar Autofocusing Methods Based on the Minimisation of the Rényi Entropy", IET Radar, Sonar and Navigation, vol. 4, no. 4, pp. 586-594, 2010.

[13] J. M. F. Moura, and Y. Jin, "Detection by Time Reversal: Single Antenna", IEEE Transactions on Signal Processing, vol. 55, no. 1, pp. 187-201, January 2007.

[14] J. M. F. Moura, Y. Jin, D. Stancil, J. Zhu, A. Cepni, Y. Jiang, and B. Henty, "Single Antenna Time Reversal Adaptive Interference Cancellation", IEEE International Conference on Acoustics, Speech and Signal Processing (ICASSP '05), vol. 4, pp. iv/1121-iv/1124, Philadelphia, March 2005.

[15] Y. Jin, and J. M. F. Moura, "Time-Reversal Detection Using Antenna Arrays", IEEE Transactions on Signal Processing, vol. 57, no. 4, pp. 1396-1414, April 2009.

[16] A. V. Oppenheim, A. S. Willsky, and S. H. Nawab, Signals and Systems, $2^{\text {nd }}$. Ed., Upper Saddle River, NJ: Prentice-Hall, 1996.

\section{VITA}

Imanol de Arriba-Ruiz was born in Donostia/San Sebastian, Spain, on February 5, 1985. He received his university degree as a Telecommunications Engineer from Basque Country University (UPV) in 2008. Since October 2008 he has been part of Microwave and Radar Group (GMR) at Signals, Systems and Radiocommunications Department (SSR) of Technical University of Madrid (UPM), where he carried out his master's degree and he is currently working towards his Ph.D. degree. His research activities and interests are focused in the area of radar images processing, both SAR (Synthetic Aperture Radar) and ISAR (Inverse SAR).

Félix Pérez-Martínez received his Ph.D. degree in Telecommunications Engineering from Technical University of Madrid (UPM) in 1982. He is Professor of Signals, Systems and Radiocommunications Department (SSR) of Technical University of Madrid (UPM) and Head of Microwave and Radar Group (GMR). His professional activities have been focused in teaching and doing research into the area of radio frequency technologies and radar systems. His work has been recognized with a high number of awards and honors. Dr. Pérez-Martínez has authored more than 200 contributions in books, scientific journals, symposium proceedings and reports, from which more than 100 are in the international area. Among others positions, Dr. Pérez-Martínez is a member both of Board of Telecommunications Engineers' Association and of Board of Directors and Executive Committee of the Circle of Technologies for Defense -in the last one he is also the Treasurer. Furthermore, he is a 
member of the Advisory Committee of BIT magazine. From February 2003 to November 2009, he was President of Committee of Technical Teaching of National Agency of Evaluation of Quality and Accreditation (ANECA) of Spanish Ministry of Education.

José María Muñoz-Ferreras received the M.Sc. and the Ph.D. degrees in electrical engineering from the Technical University of Madrid (UPM) in 2004 and 2008, respectively. Currently, he is with the Department of Signal Theory and Communications in the University of Alcalá, Madrid. His research activities are in the area of radar signal processing and advanced radar systems and concepts. Specifically, the focusing of high-resolution inverse synthetic aperture radar images and the design and validation of radar systems with the software-defined paradigm are issues of his interest. 